\title{
12.
}

\section{Notice sur un manuscrit Arabe d'un traité d'algèbre par Aboul Fath Omar Ben Ibrahîm Alkhayâmî, contenant la construction géométrique des équations cubiques.}

(Par Mr. F. Woepclé, priv. doc. à l'université de Bonn.)

I.

Les oeuvres des illustres mathématiciens, que la Grèce a produits pendant l'espace de six siècles, ont été presque continuellement l'objet de travaux savans. Dès le commencement du moyen âge, jusqu'à nos jours, elles ont été traduites, commentées, publieés, souvent par des géomètres, qui euxmêmes avaient une haute célébrité. Il suffira ici de rappeler les noms de Nassir eddin al Thusi, de Bachet de Mezériac, d'Halley.

Les découvertes importantes par lesquelles le génie Arabe pendant une période d'une semblable durée, a enrichi la même science, n'ont pas été assez heureuses pour s'attirer une pareille attention. On est même allé jusqu'à soutenir, que les Arabes n'avaient en général rien inventé, ou presque rien au delà de ce qu'ils avaient puisé des auteurs Grècs, traduits en Arabe depuis le temps des khalifes Haroun Alrachid et Almiramoun. Des recherches soigneuses et étendues meneront probablement à des résultats fort différens.

La notice présente réussira peut-être à en founir une preuve, en démontrant, que les Arabes ont systématiquement développé un problème, dont chez les Grècs on ne trouve que des traces éparses, et dont la résolution moderne est censée faire grandement honneur aux génies de Viète et de Descartes.

J'avais trouvé le traité Arabe, dont il s'agit, cité deux fois, comme contenant probablement la résolution des équations cubiques: d'abord par Montucla, ensuite, dans une dissertation de M.Garz, sur les commentateurs Arabes d'Euclide, ce qui me fit désirer de pouvoir l'examiner moi-même. M. Freitag, dont l'illustre direction m'a guidé dans l'étude de la langue Arabe, eut l'in- 
12. Woepcke, de la constr. géom. des équat. cubiques, par Alkhayâmî. 161

signe bonté de se faire envoyer en ma faveur de Leyde le volume manuscrit, dans lequel, parmi un grand nombre de mémoires, traitant de différens objets mathématiques, se trouve aussi celui $d^{\prime} A l k h a y \hat{a ̂ m} \hat{\imath}^{*}$ ).

L'identité de ce traité, ainsi que la haute estime dont il jouit chez les Orientaux, sont constatées par Hâdjî Khalfa, qui en cite le commencement. Des raisons qu'il serait trop long de détailler ici, prouvent qu'il ne fut pas écrit avant le milieu du onzième siècle, ni après la fin du quatorzième. Montucla cite un astronome Omar Cheyam, auteur de la nouvelle forme d'intercalation, introduite chez les Persans en 1079 par Gelâl eddin Melir-Shah; je n'ai pu encore vérifier s'il $\mathrm{y}$ a identité entre cet astronome et l'auteur du traité dont il s'agit.

Je vais maintenant donner un aperçu succinct de ce que ce traité contient.

II.

L'auteur commence par esquisser en quelques lignes l'histoire du problème des équations cubiques, depuis Archimède jusqu'à son propre temps; il y trouve occasion de nommer deux célèbres géomètres Arabes: Almâhâmî et Abou Djafar Alkhâzin dont d'ailleurs la mémoire nous a été conservée par le Tarikh al Hocamâ chez Casiri.

Suit une dédication au grand-juge Abou Tähir, et après cela l'introduction proprement dite du traité d'algèbre, contenant les définitions des notions fondamentales de cette science.

Ces définitions sont assez intéressantes, parcequ'elles font voir combien la philosophie d'Aristote a influé sur la science Arabe; on y reconnaît la terminologie du grand Stagirite; il s'y trouve même des passages, qui ne sont intelligibles qu'à l'aide d'une connaissances exacte de son système. Cette érudition métaphysique distingue favorablement notre auteur de Mohammed Ben Mousu, qui n'en offre point de traces.

Je remarque qu'en général on doit accorder à Alkhayâmî un rang supérieur à celui de Mohammed Ben Mousa ou de Behâ-Eddîn, vu que les traités de ceux-ci n'ont pour but que l'instruction des commençans, tandis que celui $d$ 'Alkhayamî porte un caractère plus élevé, effleurant seulement les questions d'une portée inférieure; appuyant sur les difficultés réelles, citant

*) Je ne saurais manquer de reconnaitre ici la libéralité bienveillante avec laquelle M. M. le bibliothécaire en chef et le conservateur des manuscrits de la bibliothèque de Leyde se sont prêtés à ce désir. 
les travaux des savans contemporains, corrigeant parfois leurs erreurs, ne donnant jamais des exemples en pure illustration des théorèmes proposés, à moins qu'ils n'aient une certaine célébrité historique. Ce n'est pas un des livres, qui reproduisent ce qu'on sait dans une science, mais un de ceux qui en reculent les bornes.

L'introduction de l'auteur est terminée par le tableau suivant des équations, dont il va donner la théorie.

"Equations simples."

1. $a=x$, 2. $a=x^{2}$, 3. $a=x^{3}, 4 . b x=x^{2}$, 5. $b x=x^{3}$, 6. $c x^{2}=x^{3}$, "Equations composées."

7. $x^{2}+b x=a$, 13. $x^{3}+b x=a$, 19. $x^{3}+c x^{2}+b x=a$;

8. $x^{2}+a=b x$, 14. $x^{3}+a=b x, 20 . x^{3}+c x^{2}+a=b x$,

9. $b x+a=x^{2}$,

15. $b x+a=x^{3}$,

21. $x^{3}+b x+a=c x^{2}$,

10. $x^{3}+c x^{2}=b x$,

16. $x^{3}+c x^{2}=a$,

22. $c x^{2}+b x+a=x^{3}$,

11. $x^{3}+b x=c x^{2}$,

17. $x^{3}+a=c x^{2}$,

23. $x^{3}+c x^{2}$

12. $c x^{2}+b x=x^{3}$,

18. $c x^{2}+a=x^{3}$,

24. $x^{3}+b x$

$=\boldsymbol{b} \boldsymbol{x}+\boldsymbol{a}$,

25. $x^{3}+a=c x^{2}+h x$.

Les équations $(4-6$, et $10-12$.) sont ramenées aussi par des procédés géométriques à celles qu'on en déduit en les divisant par $x$ ou $x^{2}$.

On ne trouve pas dans ce système, complet à cela près, les équatións suivantes :

$$
\begin{gathered}
x+a=0, \quad x^{2}+a=0, \quad x^{2}+b x+a=0, x^{3}+a=0, \\
x^{3}+b x+a=0, x^{3}+c x^{2}+a=0, x^{3}+c x^{2}+b x+a=0,
\end{gathered}
$$

$a, b, c$ étant positifs; ces formes impliquent un contresens pour les mathématiciens Arabes, qui regardent comme impossible la résolution d'une équation, dès qu'elles n'admet pas des racines positives; aussi cette manière d'écrire les équations, due au génie d'Harriot, où la somme de tous les termes, formant le premier membre, est égalée à zèro, est-elle essentiellement occidentale et moderne.

La résolution algébrique et géométrique des équations carrées rassemble à celle donnée par Mohammed Ben Mousa; cependant Alkhayâmî déduit sa demonstration géométrique avec beaucoup d'élégance des propositions d'Euclide: Elementa II, 5, 6, VI, 28, 29, Data 58, 59. Cette méthode a échappé à Mohammed, et pourtant celui-ci, | vivant à la cour d'Almamoun, devait connaître les Élémens d'Euclide traduits en Arabe déjà du temps de Haroun Alrachid par Hedjadj Ben Yousouf Ben Matar Alcoupihî 
III.

La résolution des équations cubiques, qui se trouve à la suite de celle des équations carrées, et qui occupe la partie principale de ce traité, n'est pas une résolution proprement dite, à la manière de celle qui porte le nom de Cardan, mais une construction géométrique; aussi l'auteur déclare-t-il exprès, qu'il lui a été impossible de les resoudre d'une autre manière et qu'il abandonne aux géomètres à venir, le problème de leur résolution algébrique.

Avant de passer à cette construction géométrique des équations cubiques, nous allons en exposer en peu de mots les principes analytiques, tels qu'ils se présentent du point de vue moderne.

Étant données $n$ équations respectivement du degré $p, q, r, \ldots w$, renfermant $n$ quantités inconnues, en èliminant entre ces équations $n-1$ inconnues, on obtiendra généralement une équation du degré $p \cdot q . r \ldots w$ qui ne renfermera qu'une seule inconnue.

Lorsque le nombre des équations données, et des inconnues qu'elles renferment, ne monte qu'à deux, leurs degrés respectifs étant $p$ et $q$, l'équation qui résulte de l'élimination, sera du degré $p . q$, et aura pour racines les valeurs des abscisses des points, où se rencontrent les deux courbes planes représentées par les équations données.

Les coefficiens de l'équation produite par l'élimination, sont des fonctions des coefficiens des équations données; on pourra donc se servir des relations qui existent entre ces deux systèmes de coefficiens, pour déterminer les coefficiens des $\boldsymbol{n}$ équations, qui renferment les $\boldsymbol{n}$ inconnues, de manière à produire par l'élimination une équation proposée, dont le degré cependant ne doit s'éléver au delà du produit $p \cdot q \cdot r \ldots w$.

Soient données les deux équations du second degré:

$$
\begin{aligned}
& \text { 1. } \alpha y^{2}+\beta y x+\gamma x^{2}+\delta y+\varepsilon x+\varphi=0, \\
& \text { 2. } \alpha \alpha_{1} y^{2}+\beta_{1} y x+\gamma_{1} x^{2}+\delta_{1} y+\varepsilon_{1} x+\varphi_{1}=0,
\end{aligned}
$$

et faisons

$$
\text { II. }\left\{\begin{array}{lll}
m_{1}=\alpha_{1} \beta-\alpha \beta_{1} & n_{1}=\beta_{1} \gamma-\beta \gamma_{1} \\
m_{2}=\alpha_{1} \gamma-\alpha \gamma_{1} & n_{2}=\beta_{1} \varepsilon-\beta \varepsilon_{1} \\
m_{3}=\alpha_{1} \delta-\alpha \delta_{1} & n_{3}=\beta_{1} \varphi-\beta \varphi_{1} & w_{1}=\delta_{1} \varepsilon-\delta \varepsilon_{1} \\
m_{4}=\alpha_{1} \varepsilon-\alpha \varepsilon_{1} & v=\gamma_{1} \delta-\gamma \delta_{1} & \\
m_{5}=\alpha_{1} \varphi-\alpha \varphi_{1} &
\end{array}\right.
$$


164 12. Wo epclee, de la constr. géom. des équat. cubiques, par Alkhayâmî.

$$
\text { II. }\left\{\begin{array}{l}
C_{4}=\left(m_{2}\right)^{2}-m_{1} n_{1} \\
C_{3}=2 m_{2} m_{4}-m_{3} n_{1}-m_{1}\left(n_{2}-v\right) \\
C_{2}=\left(m_{4}\right)^{2}+2 m_{2} m_{5}-m_{3}\left(n_{2}-v\right)-m_{1}\left(n_{3}+w_{1}\right) \\
C_{1}=2 m_{4} m_{5}-m_{3}\left(n_{3}+w m_{1}\right)-m_{1} w_{2} \\
C_{0}=\left(m_{5}\right)^{2}-m_{3} w_{2}
\end{array}\right.
$$

l'équation qu'on obtient en éliminant $y$ entre les équations (1. et 2.), sera la suivante

$$
\text { 3. } C_{4} x^{4}+C_{3} x^{3}+C_{2} x^{2}+C_{1} x+C_{0}=0 \text {. }
$$

Pour rendre l'équation (3.) identique avec une équation cubique proposée $x^{3}+c x^{2}+b x+a=0$, il suffira de déterminer les coëfficiens des équations (1. et 2.) à l'aide des rélations (I. et II.), de manière à faire évanouir $C_{4}$ et $\dot{a}$ rendre $C_{3}, C_{2}, C_{1}, C_{0}$ respectivement proportionnels $\dot{a} 1, c, b, a$.

Les équations (1. et 2.) représentent deux sections coniques; on pourra donc construire deux sections coniques, dont les points de rencontre ont pour abscisses les racines d'une équation cubique proposée; on arrivera encore à ce but, d'obtenir les racines d'une équation cubique proposée par une construction géométrique, en laissant carré-carrée l'équation, qui résulte de l'élimination, mais en déterminant les coefficiens de manière que, divisée par un de ses facteurs, elle se transforme dans l'équation cubique proposée.

Comme pour cet effet on peut disposer de 12 quantités, afin de donner à 5 fonctions de ces quantités des valeurs déterminées, on pourra satisfaire en outre à des conditions arbitraires, qui contribueront à rendre plus élégante la résolution du problème; on pourra entre autres donner à 7 coefficiens des équations (1. et 2.) les valeurs 0 ou 1 (de manière cependant à ne pas diminuer le degré de ces équations).

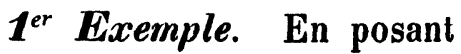

$$
\alpha=1, \quad \beta=0, \delta=0 ; \quad \alpha_{1}=0, \quad \beta_{1}=1, \quad \gamma_{1}=0, \quad \delta_{1}=0,
$$

on obtient

$$
m_{1}=-1, m_{4}=-\varepsilon_{1}, m_{5}=-\varphi_{1} ; \quad n_{1}=\gamma, \quad n_{2}=\varepsilon, \quad n_{3}=\varphi ;
$$

le reste des quantités (I.) s'évanouissent; et par suite

$$
C_{4}=\gamma, C_{3}=\varepsilon, C_{2}=\varepsilon_{1}^{2}+\varphi, C_{1}=2 \varepsilon_{1} \varphi_{1}, C_{0}=\varphi_{1}^{2}
$$

Ceux des coefficiens qui restaient d'abord indéterminés, prennent donc les valeurs suivantes: $\gamma=C_{4}, \varepsilon=C_{3}, \varepsilon_{1}= \pm \frac{C_{1}}{2 \sqrt{C_{0}}}, \varphi=C_{2}-\frac{C_{1}^{2}}{4 C_{0}}, \varphi_{1}= \pm \gamma / C_{0}$. En même temps $C_{4}=1, C_{3}=c+\xi, C_{2}=b+c \xi, C_{1}=a+b \xi, C_{0}=a \xi$ sont les coefficiens de l'équation carré-carrée, qui, divisée par le facteur $x+\xi$ 
devient identique avec l'équation cubique proposée; $\xi$ étant une quantité arbitraire, nous en disposerons à donner une forme plus élégante aux expressions que nous venons d'obtenir, ce qui se fait en posant $\xi=\frac{a}{b}$; nous aurons $C_{3}=\frac{a}{b}+c, C_{2}=b+\frac{a c}{b}, C_{1}=2 a, C_{01}=\frac{a^{2}}{b}$, d'où l'on tire $\gamma=1, \varepsilon=\frac{a}{b}+c$, $\varepsilon_{1}= \pm \sqrt{b}, \varphi=\frac{a c c}{b}, \varphi_{1}= \pm \frac{a}{\sqrt{b}}$.

\section{$\mathbf{2}^{\text {me }}$ Exemple. Posons}

$$
\beta=0, \delta=0, \varphi=0, \alpha_{1}=0, \beta_{1}=0, \varepsilon_{1}=0, \varphi_{1}=0,
$$

il vient

$$
m_{2}=-\alpha \gamma_{1}, \quad m_{3}=-\alpha \delta_{1}, v=-\gamma \delta_{1}, \quad w_{1}=\delta_{1} \varepsilon
$$

les autres quantités (I.) s'évanouissent; donc

$$
\boldsymbol{C}_{4}=\alpha^{2} \gamma_{1}^{2}, \quad \boldsymbol{C}_{3}=0, \quad \boldsymbol{C}_{2}=\alpha \gamma \delta_{1}^{2}, \quad \boldsymbol{C}_{1}=\alpha \varepsilon \delta_{1}^{2}, \quad \boldsymbol{C}_{0}=0 .
$$

Des relations $C_{3}=0$ et $C_{0}=0$ il suit, qu'en divisant par $x$ l'équation produite par l'élimination, on obtient une équation cubique, dont le second terme s'est évanoui; la combinaison choisie dans cet exemple sera donc propre à produire une équation proposée de la forme $x^{3}+b x+a=0$. Posons pour cet effet $b=\frac{\gamma \delta_{1}^{2}}{\alpha \gamma_{1}^{2}}, a=\frac{\varepsilon \delta_{1}^{2}}{\alpha \gamma_{1}^{2}}$; on peut disposer de 5 quantités pour en déterminer deux autres, ce qui permet de donner à trois des premières des valeurs arbitraires; posons donc $\alpha=1, \gamma=1, \gamma_{1}=1$, il vient $\delta_{1}= \pm \sqrt{ } b$, $\varepsilon=\frac{a}{b}$.

\section{$3^{\text {me }}$ Exemple. Posons}

$$
\alpha=0, \gamma=0, \delta=0, \varepsilon=0, \beta_{1}=0, \gamma_{1}=0, \delta_{1}=0,
$$

on aura

$$
m_{1}=\alpha_{1} \beta, \quad m_{5}=\alpha_{1} \varphi, n_{2}=-\beta \varepsilon_{1}, n_{3}=-\beta \varphi_{1}
$$

les autres quantités (I.) s'évanouissent; par suite on a

$$
C_{4}=0, C_{3}=\alpha_{1} \beta^{2} \varepsilon_{1}, \quad C_{2}=\alpha_{1} \beta^{2} \varphi_{1}, C_{1}=0, C_{0}=\alpha_{1}^{2} \varphi^{2} .
$$

De ce que $C_{4}=0$, on voit qu'on arrive directement à une équation cubique, dont, parceque $C_{1}=0$, le troisième terme s'évanouit; la combinaison adoptée ici produira donc une équation de la forme $x^{3}+c x^{2}+a=0$, dès qu'on aura fait $c=\frac{\Phi_{1}}{\varepsilon_{1}}, a=\frac{\alpha_{1} \varphi^{2}}{\beta^{2} \varepsilon_{1}}$. Comme dans l'exemple précédent, on pourra déterminer arbitrairement trois de ces quantités; faisons $\alpha_{1}=1, \beta=1, \varepsilon_{1}=m$, il vient $\varphi= \pm \sqrt{a} . m, \varphi_{1}=m . c$.

Nota. La plus simple valeur à donner à $\varepsilon_{1}$, aurait été évidemment 1; mais sans doute on s'est aperçu déjà que les combinaisons que je viens de proposer, Crelle's Journal f. d. M. Bd. XL. Heft 2. 
ne sont pas choisies au hasard, mais qu'elles ont un certain rapport à la méthode dont se sert l'auteur Arabe; c'est aussi la raison pourquoi $\varepsilon_{1}$ est laissé indéterminé ici. On sait d'ailleurs qu'il est regardé comme une faute, de construire une équation cubique par l'intersection de deux sections coniques; cette construction pouvant s'opérer plus simplement.

\section{IV.}

Je ne crois pouvoir mieux caractériser à présent la méthode $d^{\prime}$ Alkhayámî qu'en traduisant d'abord sa résolution d'une des équations dont il s'occupe, pour exposer ensuite les traits généraux de ses constructions; j'accompagnerai cette traduction d'un commentaire ${ }^{*}$ ); rendant par les notations modernes le procédé du géomètre Arabe. (Pl. II.)

„La cinquième espèce des équations cubiques à trois termes est la suivante: un cube et un nombre sont égaux à des carrés. Faisons la ligne $\boldsymbol{A C}$ égale au nombre des carrés, et formons un cube égal au nombre donné, et dont le côté soit $\boldsymbol{H}$; la ligne $\boldsymbol{H}$ doit nécessairement être, ou égale à la ligne $\boldsymbol{A C}$, ou plus grande que $\boldsymbol{A C}$, ou plus petite que $\boldsymbol{A C}$. Lorsque les deux lignes sont égales, la résolution est impossible, parceque le côté du cube cherché

*) No. 17. $x^{3}+a=c x^{2} \ldots a, c, x$ désignent des quantités positives.

$$
A C=c, H^{3}=a \cdot H \gtreqless A C \text {. }
$$

I. $H=A C \ldots x \overline{\bar{F}} H ;$ 1. $x=H \ldots A C \cdot x^{2}=H^{3}$ ou $c x^{2}=a$, donc $c x^{2}<a+x^{3}$, 2. $x<H \ldots A C \cdot x^{2}<H^{3}$ ou $c x^{2}<a$, donc $c x^{2}<a+x^{3}$, 3. $x>H \ldots x^{3}>A C . x^{2}$ ou $x^{3}>c x^{2}$, donc $x^{3}+a>c x^{2}$.

II. $H>A C \ldots$ impossible par les mêmes raisons.

III. $\mathrm{H}<A C, B C=H \ldots B C \sum A B$ ou $\sqrt[3]{ } a \bar{\sum}_{\frac{1}{2}} c$.

(Observons encore en passant que dans le manuscrit Arabe les figures, contrairement à leur distination ordinaire, ne semblent être ajoutées au texte, que pour en embrouiller encore le sens, obscurci par de nombreuses fautes de copie.)

BCDE ... carré $=H^{2}=\epsilon^{\frac{2}{3}} ; D R, D T \ldots$ hyperbole équilatère, dont $C A, C E$ les asymptotes; $A T, A L, A K \ldots$ parabole, dont $B C$ le parametre. (Pour les propriétés des sections coniques employées dans ses démonstrations, Alkhayûmî a renvoyé préalablement aux deux premiers livres des sections coniques d'Apollonitus.)

1) $B C=A B \ldots B D^{2}=A B . B C$, donc $D$ un point qui satisfait à l'équation de la parabole; l'autre point dont parle l'auteur, aura pour abscisse (à compter de $C$ ) $x=\frac{1}{4} c\{1+\sqrt{ } 5\}$ et pour ordonnée $y=\{\sqrt{ } 5-1\} \frac{1}{4} c$.

2) $B C>A B \ldots B D^{2}>A B . B C$, d'où il suit en effet que la parabole passe en deçà du point $D$. L'auteur dit encore que lorsque $\sqrt[3]{ } a>\frac{1}{2} c, x$ doit être compris entre $c$ et $\sqrt[3]{a}$; de l'équation proposée $x^{3}+a=c x^{2}$ il suit immédiatement $c x^{2}>x^{2}$ ou $x<c$; il reste donc à prouver que $x>\sqrt[3]{a}$. Observons d'abord qu'il ne pourra 
doit nécessairement être, ou égal à $\boldsymbol{H}$, ou plus grand que $\boldsymbol{H}$, ou plus petit que $\boldsymbol{H}$. Lorsqu'il est égal à $\boldsymbol{H}$, son carré, multiplié par $\boldsymbol{A C}$, sera égal au cube de $\boldsymbol{H}$; donc le nombre sera égal au nombre des carrés, sans qu'on ait besoin d'y ajouter encore le cube. Lorsque le côté cherché est plus petit que $\boldsymbol{H}$, son carré, multiplié par $\boldsymbol{A C}$, sera plus petit que le nombre donné: donc le nombre donné de carrés sera déjà plus petit que le nombre donné, sans ajouter encore quelque chose à ce dernier. Lorsque le côté est plus grand que $\boldsymbol{H}$, son cube sera plus grand que son carré, multiplié par $A C$, sans qu'on ajoute au premier le nombre donné."

"Ensuite lorsque $\boldsymbol{H}$ est plus grand que $\boldsymbol{A C}$, l'impossibilité aura lieu dans les trois cas, à plus forte raison; il faut donc que $\boldsymbol{H}$ soit plus petit que $\boldsymbol{A C}$; si non, la résolution est impossible."

„Or retranchons de $A C$ la ligne $B C$, égale à $H$; la ligne $B C$ sera, ou égale à $\boldsymbol{A B}$, ou plus grande, ou plus petite. Qu'elle soit dans la première figure égale, dans la seconde plus grande et dans la troisième plus petite; formons dans les trois figures le carré $\boldsymbol{D C}$, et faisons passer par le point $\boldsymbol{D}$ une hyperbole, dont $\boldsymbol{C A}, \boldsymbol{C E}$ soient les asymptotes; ce sera dans la première figure la courbe $\boldsymbol{D R}$ et dans les deux autres $\boldsymbol{D T}$. Décrivons en outre une

être question d'une rencontre des deux sections coniques, que tant que $\sqrt[3]{u}<\frac{2}{3} c$, parceque de $\sqrt[3]{a} \equiv \frac{2}{3} c$ il suit $27 a \equiv 8 c^{3}>4 c^{3}$, ce qui rendrait l'intersection imaginaire. Ensuite, désignant $c x^{2}-x^{3}$ par $f(x)$, on aura $\frac{d f(x)}{d x}=3 x\left\{\frac{2}{3} c-x\right\}$, d'où il suit que pour toutes les valeurs de $x$, comprises entre 0 et $\frac{2}{3} c, f(x)$ décroîtra avec $x$. Pour $x=\sqrt[3]{a}$, puisque en même temṕs $c<2 \sqrt[3]{ } a$, on trouve $c x^{2}-x^{3}<a$; donc pour toutes les valeurs de $x$ plus petites que $\sqrt[3]{ } a, c x^{2}-x^{3}<a$, ce qu'il s'agissait de prouver. Le cas du contact donne deux racines égales et positives $x=\frac{2}{3} c$.

3) $B C<A B ; B D^{2}<A B . B C \ldots$ La parabole passe au delà du point $\boldsymbol{D}$ et doit nécessairement rencontrer l'hyperbole en deux points, ce qu'on trouve aussi en deduisant de $\sqrt[3]{ } a<\frac{1}{2} c \ldots 4 c^{3}>32 a>27 a$ :

$T R \perp A C, T R \perp E C$, l'hyperbole fait $R C: B C=B C: T R$

d'où suit

$$
\text { la parabole } B C: T R=T R: R A,
$$

$$
\begin{aligned}
R C^{2}: B C^{2}=B C: R A \ldots B C^{3}=R C^{2} . R A \text { ou } a & =R C^{2} \cdot R A, \\
a+R C^{3} & =R C^{2} \cdot R A+R C^{3}=R C^{2} . A C, \\
\text { donc } a+R C^{3} & =R C^{2} . c \ldots x=R C .
\end{aligned}
$$

Quant aux cas particuliers et aux problèmes impossibles, dont parle l'auteur, en voici la discussion moderne: l'équation $x^{3}-c x^{2}+a=0$ admet toujours une racine négative (de laquelle le mathématicien Arabe ne s'occupe point), et ses deux autres racines sont positives ou imaginaires selon que $27 a \lessgtr 4 c^{3}$. 
parabole dont le sommet soit $\boldsymbol{A}$, l'axe $\boldsymbol{A C}$ et le paramètre $\boldsymbol{B C}$; ce sera dans la première figure $\boldsymbol{A T}$, dans la seconde $\boldsymbol{A L}$, dans la troisième $\boldsymbol{A K}$. Les deux sections coniques seront connues de position. Dans la première figure la parabole passera par le point $\boldsymbol{D}$, parceque le carré de $\boldsymbol{B D}$ est égal au produit de $\boldsymbol{A B}$ par $\boldsymbol{B C}$; donc le point $\boldsymbol{D}$ sera situé sur la périphérie de la parabole; cetle dernière rencontrera l'hyperbole encore dans un autre point; ce que la moindre réflexion vous fera reconnaître. Dans la seconde figure le point $\boldsymbol{D}$ sera situé en dehors de la périphérie de la parabole, parceque le carré de $\boldsymbol{B D}$ sera plus grand que le produit de $\boldsymbol{A B}$ par $\boldsymbol{B C}$; alors, si les deux sections coniques se rencontrent dans un autre point pour se toucher ou s'entrecouper (ce qui fait que la perpendiculaire menée de ce point rencontre nécessairement la ligne $\boldsymbol{A C}$ entre les points $\boldsymbol{A}$ et $\boldsymbol{B}$ ), la résolution sera possible; si non, elle sera impossible. Ce contact, ou cette intersection, ont échappé à l'excellent géomètre Aboul Djoud; il déclara donc la résolution impossible, dès que $\boldsymbol{B C}$ est plus grand que $\boldsymbol{A B}$; en quoi il s'est trompé. Cette espèce d'équation est aussi celle parmi les six espèces, dont avait besoin Alkhayâmâ. Ainsi remarquez-la. Dans la troisième figure le point $\boldsymbol{D}$ est situé dans l'intérieur de la parabole, et les deux sections coniques se rencontrent en deux points."

„Dans tous les cas, menons du point de rencontre une perpendiculaire à la ligne $\boldsymbol{A B}$; dans la seconde figure ce sera $\boldsymbol{T R}$; puis une seconde perpendiculaire du même point à la ligne $C E$, ce sera $T K$; les aires $T C$ et $D C$ sont égales, donc $\boldsymbol{R} C$ à $\boldsymbol{B C}$ comme $\boldsymbol{B C}$ à $\boldsymbol{T R}$; en même temps, parceque TR est ordonnée de la section conique $A T L$, son carré sera égal au produit $\boldsymbol{A R} . \boldsymbol{B C}$, d'où l'on tire: $\boldsymbol{B C}$ à $\boldsymbol{T R}$ comme $\boldsymbol{T R}$ à $\boldsymbol{R A}$; donc on a quatre lignes en proportion continue: $\boldsymbol{R C}$ à $\boldsymbol{C B}$ comme $\boldsymbol{B C}$ à $\boldsymbol{T R}$, et comme $\boldsymbol{T R}$ à $R A$; et parsuite le carré de $R C$ au carré de la seconde $B C$, comme la seconde $\boldsymbol{B C}$ à la quatrième $\boldsymbol{R A}$. Il suit de cela que le cube de $\boldsymbol{B C}$, qui est égal au nombre donné, doit être égal au solide dont la base est le carré de $\boldsymbol{R C}$, et la hauteur $\boldsymbol{R A}$; ajoutons à tous les deux le cube de $\boldsymbol{R C}$, il suit que le cube de $R C$, ensemble avec le nombre donné, est égal au solide dont la base est le carré de $R C$, et la hauteur $A C$, qui était égale au nombre donné des carrés; c'est là ce qu'il s'agissait de trouver."

„Or nous venons de démontrer, que cette espèce d'équations cubiques comprend différens cas particuliers et qu'elle renferme des problèmes impossibles; la résolution a été effectuée par la combinaison des propriétés de deux sections coniques, d'une parabole et d'une hyperbole." 
12. Wocpcke, de la constr. géom. des équat. cubiques, par Alkhayâmî.

V.

D'une manière analogue à celle-ci, Alkhayâmî construit, une à une, toutes ses équations cubiques; ce sont les suivantes
3. $x^{3}-a=0$
19. $x^{3}+c x^{2}+b x-a=0$
13. $x^{3}+b x-a=0$
20. $x^{3}+c x^{2}-b x+a=0$
14. $x^{3}-b x+a=0$
21. $x^{3}-c x^{2}+b x+a=0$
15. $x^{3}-b x-a=0$
22. $x^{3}-c x^{2}-b x-a=0$
16. $x^{3}+c x^{2}-a=0$
23. $x^{3}+c x^{2}-b x-a=0$
17. $x^{3}-c x^{2}+a=0$
24. $x^{3}-c x^{2}+b x-a=0$
18. $x^{3}-c x^{2}-a=0$
25. $x^{3}-c x^{2}-b x+a=0$.

En déduisant les équations analytiques des sections coniques, dont il se sert dans ces constructions, et en comparant ensuite entre elles ces équations, on arrive à un résultat assez intéressant, dont voici l'exposé.

Désignons par $p, q, r, s, t$ des quantités qui ne peuvent prendre que les valeurs +1 ou -1 , ce qui permettra de poser $p q=\frac{p}{q}, p^{2}=1$, etc. la méthode du géomètre Arabe se reduit aux trois systèmes suivants:

I. $\quad y^{2}+p x^{2}+q \frac{a}{b} x=0\left\{\begin{array}{l}p=0 \ldots \text { parabole } \\ p=+1 \ldots \text { cercle } \\ p=-1 \ldots \text { hyperbole }\end{array}\right.$

$$
\frac{x^{2}-\sqrt{b} \cdot y=0}{x^{4}+p b x^{2}+q a x=0} \text { ou } x^{3}+p b x+g a=0 ;
$$

II.

$$
\begin{aligned}
& \boldsymbol{y} \boldsymbol{x}-\sqrt{ } \boldsymbol{a} \cdot \boldsymbol{m}=\mathbf{0} \ldots \text { hyperbole }
\end{aligned}
$$

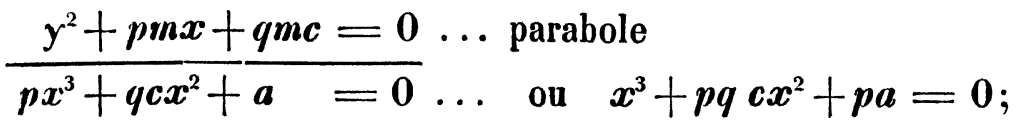

III.

$$
\begin{aligned}
& y^{2}+p x^{2}+q\left\{\frac{a}{b} \pm c\right\} x+r \frac{a c}{b}=0\left\{\begin{array}{l}
p=+1 \ldots \text { cercle } \\
p=-1 \ldots \text { hyperbole }
\end{array}\right. \\
& y x+s \sqrt{b} \cdot x+t \frac{a}{\sqrt{b}}=0 \ldots \text { hyperbole }
\end{aligned}
$$

$$
p x^{4}+q\left\{\frac{a}{b} \pm c\right\} x^{3}+\left\{b+r \frac{a c}{b}\right\} x^{2}+2 \text { st } a x+\frac{a^{2}}{b}=0
$$

ou $x^{4}+p q\left\{\frac{a}{b} \pm c\right\} x^{3}+p\left\{b+r \frac{a c}{b}\right\} x^{2}+2 p s t a x+p \frac{a^{2}}{b}=0$.

A l'aide du système $I$. peuvent être construites les équations $(3,13,14,15)$ lorsqu'on pose 
19. Woepclie, de la constr. géom. des équat. cubiques, par Alkhayâmî.

$$
\begin{array}{rll}
\text { 3. } & p=0 & q=-1 \quad b=1 \\
\text { 13. } & p=+1 & q=-1 \\
\text { 14. } & p=-1 & q=+1 \\
\text { 15. } & p=-1 & q=-1 ;
\end{array}
$$

à l'aide du système II. les équations $(16-18)$ lorsqu'on fait

$$
\begin{array}{llll}
\text { 16. } & m=\sqrt{ } a & p=-1 & q=-1 \\
\text { 17. } & m=\sqrt[3]{ } a & p=+1 & q=-1 \\
\text { 18. } & m=c & p=-1 & q=+1 ;
\end{array}
$$

enfin le système III. répond aux équations (19-25) lorsqu'aux quantités $p, q, r, s, t$ on donne les valeurs suivantes:

$$
\begin{array}{lrllllll} 
& 19 . & 20 . & 21 . & 22 . & 23 . & 24 . & 25 . \\
p & +1 & -1 & +1 & -1 & -1 & +1 & -1 \\
q & -1 & +1 & +1 & -1 & -1 & -1 & +1 \\
r & -1 & +1 & -1 & +1 & -1 & +1 & -1 \\
s & +1 & -1 & -1 & -1 & -1 & -1 & -1 \\
t & -1 & +1 & -1 & -1 & -1 & +1 & +1
\end{array}
$$

On ramène l'équation carré - carrée qui résulte du système III, à l'équation cubique proposée $x^{3}+u c x^{2}+v b x+w a=0$ en la divisant par $\left(x \pm \frac{a}{b}\right)$, ce qui peut être démontré aisément comme suit:

$$
\begin{gathered}
\left\{x^{3}+u c x^{2}+v b x+w x\right\} \cdot\left\{x+\frac{w}{v} \cdot \frac{a}{b}\right\} \\
=x^{4}+\left\{u c+\frac{w}{v} \cdot \frac{a}{b}\right\} x^{3}+\left\{v b+\frac{u w}{v} \cdot \frac{a c}{b}\right\} x^{2}+2 w a x+\frac{w^{2}}{v} \cdot \frac{a^{2}}{b} \\
=x^{4}+\left\{v w \frac{a}{b}+u c\right\} x^{3}+v\left\{b+u w \frac{a c}{b}\right\} x^{2}+2 w a x+v \frac{a^{2}}{b},
\end{gathered}
$$

où les valeurs à donner aux quantités $u, v, w$ sont les suivantes:

19. 20. 21. 22 . 23. 24. 25.

$$
\begin{array}{cccccccc}
u & +1 & +1 & -1 & -1 & +1 & -1 & -1 \\
v & +1 & -1 & +1 & -1 & -1 & +1 & -1 \\
w & -1 & +1 & +1 & -1 & -1 & -1 & +1
\end{array}
$$

On voit maintenant que c'est exactement la méthode qui, dans les trois exemples proposés ci-dessus, a été déduite des principes analytiques de la construction géométrique des équations algébriques.

Je remarque encore qu'Alkhayâmî̀ semble avoir ignoré, que dans l'équation générale du troisième degré on peut toujours faire disparaître le second terme, ce qui rend superflus les systèmes II et III. 
12. Wocpcke, de la constr. géom. des équat. cubiques, par Alkhaydmî.

VI.

Il me reste à parler du nombre des racines que l'auteur trouve pour chacune de ces équations.

Observons en premier lieu que les racines négatives ou imaginaires n'ont pas d'existence pour l'algébriste Arabe; il ne s'occupe donc que des racines positives, et dès qu'un problème n'en admet pas, il en regarde la résolution comme impossible.

Donc celle des trois racines de l'équation cubique, qui est toujours réelle et dont le signe est opposé à celui du terme constant de l'équation, n'est remarquée par Alkhayâmî, que lorsqu'elle est positive; c'est à dire dans les équations $(3,13,15,16,18,19,22,23,24)$.

Relativement aux deux autres racines qui sont réelles ou imaginaires selon la nature des coefficiens de l'équation, Alkhayâmê découvre le véritable critérium géométrique de leur réalité: la rencontre en deux points, ou le contact des deux sections coniques, dont la combinaison représente l'équation proposée.

Dans un procédé purement géométrique, on ne doit pas s'attendre à trouver établie aussi la relation algébrique entre les coefficiens, qui répond au critérium énoncé; cependant on vient d'observer dans l'exemple traduit, qu'Aboul Djoud avait essayé de la trouver; malheureusement il était tombé dans des erreurs, que le génie pénétrant d'Alkhayâmî ne manque pas de relever.

Je remarque en passant, que les deux racines dont il s'agit, sont toujours imaginaires dans les cas $(3,13,18$.$) . Elles sont positives ou imaginaires$ dans les cas $(14,17,20,21,24,25)$. Dans tous les autres cas elles sont négatives ou imaginaires.

Au cas du contact des deux sections coniques, Alkhayámî̀ ne reconnait pas encore dans la valeur obtenue deux racines, égales seulement en quantité, mais essentiellement différentes.

Quant aux racines positives, Alkhayâmı̂ discute soigneusement, et à deux erreurs près, avec une justesse parfaite le nombre des points de rencontre des deux sections coniques (du côté des coordonnées positives); ce qui revient à une détermination du nombre des racines positives. Voici ces deux erreurs. En discutant l'équation $x^{3}-c x^{2}+b x-a=0$, il ne remarque pas, qu'elle peut avoir trois racines positives et n'a égard qu'à celle des trois racines, qui est toujours positive; puis dans l'équation $x^{3}-c x^{2}-b x+a=0$, 
en discutant séparément le cas $\frac{a}{b} \geqq c$, pendant que $\frac{a}{b} \leqq c$, il ne parle que d'une seule des racines positives, tandis qu'il y en a deux.

Malgré le génie extraordinaire qu'on ne saurait lui disputer, Alkhayâma n'a donc point reconnu cette vérité fondamentale, que toute équation cubique a essentiellement trois racines.

\section{VII.}

A la construction des équations cubiques se trouve jointe, en guise de corollaire, la partie de ce traité la plus importante pour la connaissance du point de vue, où se place l'auteur vis-à-vis des équations algébriques en général.

$\boldsymbol{A}$ lkhayâmî̀ y discute les équations aux puissances négatives de l'inconnue, ou renfermant en même temps des puissances négatives et positives de l'inconnue, dont la résolution peut être ramenée à celle des équations carrées et cubiques.

Alkhayâanî y comprend les équations binomes du quatrième et du sixième degré. Relativement à celle du cinquième degré, il observe que sa résolution ne peut être réduite aux méthodes exposées dans ce traité (ce qui en effet ne peut se faire), mais il renvoie pour ce problème à la solution (géométrique) qu'il dit en avoir été donnée par Abou Ali lbn Ahä̈tam, célèbre géomètre Arabe, sur la vie et les écrits duquel on trouve des détails intéressants dans le Tarikh al Hocamâ chez Cusiri.

Aux 25 équations, dont la discussion occupe la partie principale de ce traité, Alkhayâmî, de.cette manière, en ajoute 61 autres. Après en avoir fini la discussion, l'auteur se résume en ces termes:

„La totalité des équations ayant lieu entre ces sept degrés*), et résolubles par les méthodes que nons venons d'exposer, montè donc à quatrevingt-six espèces, dont les traités de mes prédecesseurs ne contiennent que six."

Les six espèces dont veut parler l'auteur sont évidemment les suivantes:
1. $a=b x$,
2. $a x=b x^{2}$,
3. $a=b x^{2}$,
4. $a=b x+c x^{2}$,
5. $a x=b+c x^{2}$,
6. $a x^{2}=b+c x$,

dont la résolution embrasse toute l'algèbre, également de Mohammed Ben Mousa et de Behâ-Eddin. Il parait donc, qu'au temps du second on avait oublié déjà une partie de ce qu'au temps du premier on ne savait pas encore.

*) Savoir les équations renfermant les sept puissances suivantes de l'inconnue:

Bonn, au mois de Mars 1850.

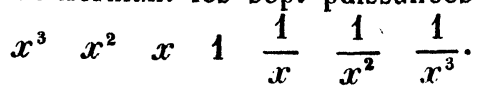

